\title{
TITLE: The Incidence of Postoperative Complications in the PACU
}

\author{
AUTHORS: Kelly V. Mayson FRCPC, Joanne E. Beestra BSN, Peter T. Choi FRCPC \\ AFFLIATION: Department of Anesthesia, Vancouver General Hospital, University of British \\ Columbia, Vancouver Hospital
}

INTRODUCTION: Over the past 10 years, an emphasis on decreasing postoperative complications (POC) has led to increased use of pre-emptive analgesics, prophylactic antiemetics, and newer short-acting anesthetic agents. Most estimates of the incidence of POC are based on data from the late 1980's and early 1990's. To established a baseline estimate for future quality assurance projects, we determined the current inhospital incidence of POC at a large tertiary care teaching hospital.

METHODS: A database of all postoperative patients admitted to the postanesthetic care unit (PACU) at the Vancouver General Hospital was reviewed to establish the inhospital incidence of POC from April 2001 to March 2004 using the ORMIS program. We included data from all adult patients undergoing elective or emergent general, gynecological, ophthalmological, major orthopedic, otorhinolaryngological, neurosurgical, plastic, thoracic, urological, or vascular procedures. Data from direct admissions to the intensive care unit were excluded. A standardized form with predefined complications was completed by the PACU nursing staff and reviewed by a nurse clinician. Common complications were then examined in more detail with respect to their type of surgery. Descriptive statistics were used.

RESULTS: We analyzed data from 37,071 patients. Respiratory complications (all combined, $15.2 \%$ ), cardiovascular complications (all combined, $12.3 \%$ ), postoperative nausea and vomiting (PONV, 9.4\%), and excessive pain (7.2\%) were common complications. Respiratory complications included inability to extubate immediately after surgery $(5.8 \%)$, desaturation (2.1\%), and hypoventilation (2\%). The most common cardiovascular complications were hypotension (4.5\%), hypertension (4.3\%), and dysrhythmias (2.8\%).

The incidence of PONV was highest after gynecological procedures (15.5\%; 616/3972), followed by spine $(12.96 \% ; 250 / 1929)$, reconstructive orthopedics $(11.9 \% ; 356 / 2997)$, and general surgerical procedures $(10.9 \% ; 678 / 6233)$. Incidence of excessive pain was highest after spine $(12.3 \% ; 238 / 1929)$, thoracic $(10.36 \% ; 256 / 2470)$, gynecological $(9.6 \% ; 381 / 3972)$, and general surgical procedures $(8.9 \% ; 555 / 6233)$.

DISCUSSION: Despite pharmacological and medical aptitude advances in the past 10 years, our incidences of POC such as PONV, hypotension, and dysrhythmias remain similar to estimates from 1992(1). We are now attempting to target our two most common POC, pain and PONV, with quality assurance projects focusing on the introduction of clinical guidelines on the management of these problems.

REFERENCES: (1) Hines $R$, et al. Complications occurring in the postanesthesia care unit: a survey. Anesth Analg 1992:74:503-9. 